\title{
Quasi-Passive and Reconfigurable Optical Node: Implementations with Discrete Latching Switches
}

\author{
J. Jin ${ }^{(1)}$, Y. Bi ${ }^{(1)}$, M. De Leenheer ${ }^{(1,2)}$, L. G. Kazovsky ${ }^{(1)}$, J. P. K. Perin ${ }^{(3)}$, M. R. N. Ribeiro ${ }^{(3)}$ \\ ${ }^{(1)}$ Stanford University, USA ${ }^{(2)}$ Ghent University - IBBT, Belgium ${ }^{(3)}$ Federal University of Espirito Santo, Brazil \\ Email: jingjin@stanford.edu
}

\begin{abstract}
Quasi-Passive and Reconfigurable (QPAR) optical nodes are implemented using two different discrete optical latching switches based on Micro-Opto-Mechanical and Magneto-Optic principles. A clear trade-off between speed and power consumption is noticed for those QPAR realizations.
\end{abstract}

Keywords - quasi-passive optical network; latching switch; energy efficiency

\section{INTRODUCTION}

To better accommodate the increasing bandwidth requirement in today's networks, the fiber optics should be laid as close as possible, ideally right into the subscribers' houses. To realize this concept of Fiber-To-The-x (FTTx), Passive Optical Networks (PONs) are widely deployed. In the legacy Time Division Multiplexing (TDM) PONs, passive optical splitters are used to broadcast the signal to all the customers in a Pointto-Multiple-Point (P2MP) manner. Because of the rigid power splitting ratio, PONs can only connect subscribers who are geographically close $(10 \sim 20 \mathrm{~km})$ to the Central Office $(\mathrm{CO})$. In addition, because the bandwidth of a PON is shared among subscribers in the whole tree structure, the bandwidth-per-user is limited by the splitting ratio and individual bandwidth upgrades are virtually precluded [1]. As a future-proof technology of FTTx, Active Optical Networks (AONs) have better performance in those aspects. In AONs, signals are directed to specific customers in a Point-to-Point (P2P) manner by electrically powered switching equipment, such as a router or a switch/aggregator. By introducing the flexibility through switches, AONs extend the maximum range to $70 \mathrm{~km}$ without repeaters and enable the possibility for pay-as-yougrow bandwidth upgrade. However, AONs nodes are composed of moving and electrical parts, and as such have high-energy consumption, high maintenance costs and lower reliability.

In response, Quasi-Passive And Reconfigurable (QPAR) optical nodes are proposed to trade off among range, bandwidth upgradability and power consumption [2]. The quasi-passive feature implies power is only consumed during reconfiguration of the node, and is based on latching switches. In this paper, QPAR nodes are experimentally demonstrated and characterized based on two alternative physical principles for latching mechanisms, namely Micro-Opto-Mechanical and Magneto-Optic.

\section{QPAR IMPLEMENTATION WITH DISCRETE SWITCHES}

The three major functions of the QPAR node [3] are: (i) extending the network range by splitting the input power into various levels according to the actual geographic distribution of subscribers; (ii) realizing individual bandwidth upgrade by allocating additional wavelengths to selected subscribers; (iii) achieving bandwidth upgrade and power splitting ratio flexibility at low power consumption.

Although QPAR was originally designed to use integrated optical devices, this paper evaluates how discrete elements can be used to perform the basic tasks of QPAR with the help of optical latching switches (OLSs). The proposed $2 \times 3 \times 2$ (two wavelengths, two possible output power levels, two outputs) QPAR structure is illustrated in Fig. 1.

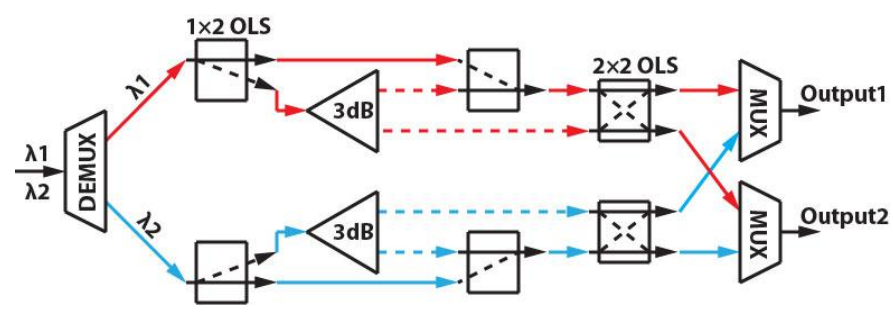

Fig. $12 \times 3 \times 2$ QPAR implemented with discrete elements.

\section{OPTICAL LATCHING SWITCHES}

OLSs are indispensible to realize the quasi-passive operation. A wide variety of optical switches based on different latching mechanisms is available: (1) Micro-Opto-Mechanical Systems (MOMS) based [4], (2) Magneto-Optic (MO) effect based [5], (3) Transition metal oxide based [6], and (4) Ferroelectric Liquid Crystal (FLC) based [7].

Among these mechanisms, Transition Metal Oxide and FLC based switches can latch states with high efficiency. The manufacturing process, however, is immature and difficult to be integrated with current CMOS technology. In contrast, the MOMS and MO effect based latching switches are well studied, cost-efficient and therefore widely used. Pairs of suspension beams mechanically latch the MOMS-OLS, whereas the MO-OLS is based on the Faraday effect in ferromagnetic material. In our research, two commercially available MOMS [4] and MO effect based [5] latching switches are compared. 


\section{A. Requirements of OLSs in QPAR}

Insertion loss: Most PON vendors adopt a Class $\mathrm{B}+$ transceiver with $28 \mathrm{~dB}$ optical path loss. Considering legacy PON with $20 \mathrm{~km}$ reach, $0.35 \mathrm{~dB} / \mathrm{km}$ fiber loss and $3 \mathrm{~dB}$ connector loss, each optical path of QPAR should have no more than $18 \mathrm{~dB}$ insertion loss. Therefore, the insertion loss of a single OLS should be small enough so that the sum does not exceed this power margin while being cascaded in QPAR.

Switching time: Switch reconfiguration is required during network deployment changes or when working wavelengths are added or dropped. Therefore, switching time should be smaller than the traffic restoration time, which is usually in the order of a few tens of milliseconds [8].

Energy consumption: Remotely feeding the QPAR with the needed energy to enable state changes will be a challenge. Low power can be transported optically from the $\mathrm{CO}$ and locally stored, so OLSs used in QPAR should have minimal power consumption to allow larger number of operations of QPAR nodes.

\section{DEMONSTRATION AND CHARACTERIZATION}

The $2 \times 3 \times 2$ QPAR node functionality is successfully demonstrated in Fig. 2 (a), which shows all the possible states of the MOM-OLSs based QPAR. It is clear from Fig. 2 (b) that optical power delivered to a given output can be adjusted from none, half, and full power per wavelength.

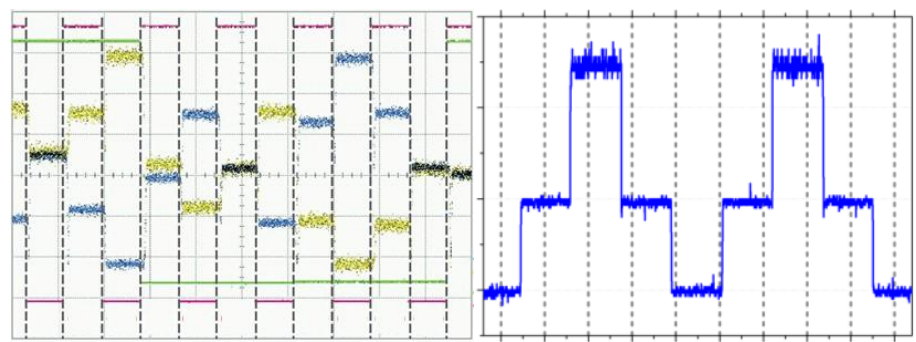

(a)

(b)

Fig. 2 (a) Electric control signals (Pink and Green) and optical signals (Blue and Yellow) at both output ports for MOM-OLSs based $2 \times 3 \times 2$ QPAR. (b) Output optical signal at one output ports for MO-OLS based $1 \times 3 \times 2$ QPAR (electric control signals not shown)

Table 1 compares the performance of QPAR using different OLSs. Besides the coupling loss with the optical fiber in both cases, the loss caused by the imperfection of the vertical micro-mirror in MOM-OLS is less significant than the absorption loss in the ferromagnetic material of MO-OLS. Differences in path loss can influence the actual power level delivered, as can be seen in Fig.2 (b). The crosstalk of QPAR is mainly affected by the OLS closest to the output port. For a single switch, limited by the Polarization Extinction Ratio (PER) of PBS in MO-OLS, its crosstalk is lower than that of MOM-OLS. During reconfiguration, all the OLSs operate in parallel, resulting in a roughly identical switching time for a single switch and the full QPAR node. Mechanical switching is in general slower because of inertia, and this is demonstrated in our experiments. However, due to its inductive nature, MO-OLSs have considerably higher power consumption than MOM-OLSs.

Table 1 Performance of QPAR nodes.

\begin{tabular}{|c|c|c|}
\hline Parameter & MOM-OLS & MO-OLS \\
\hline Insertion loss $(\mathrm{dB})$ & 5 & 6.5 \\
\hline Crosstalk $(\mathrm{dB})$ & 72 & 50 \\
\hline $\begin{array}{c}\text { Switching Time }(\mu \mathrm{s}) \\
\text { Power consumption }(\mathrm{mW})\end{array}$ & $400-600$ & $20-70$ \\
(for continually changing states) & 30 & 450 \\
\hline
\end{tabular}

\section{CONCLUSION}

We presented an experimental demonstration of QPAR based on Micro-Opto-Mechanical and Magneto-Optic latching switches. MOM-OLSs bring lower insertion loss and larger crosstalk, while MO-OLSs offer faster response time but at the expense of higher levels of power consumption. Further studies will include investigations on the stability of latched states in both architectures as well as more economic approaches to QPAR node architectures.

\section{ACKNOWLEDGMENT}

We would like to thank Sercalo Microtechnology and Neophotonics Corporation for sponsoring this work and FAPES for funding the MO-OLS based QPAR (Grant 45445648).

\section{REFERENCES}

[1] KEYMILE Report, "AON vs. PON - A comparison of two optical access network technologies and the different impact on operations," KEYMILE International GmbH, 2008.

[2] S. Yen, M. Tendulkar, J. R. Jameson, S. Yamashita, Y. Nishi, O Solgaard, and L. G. Kazovsky, "Quasi-Passive and Reconfigurable Node for Optical Access Network," in Proc. Conference on Lasers and Electro-Optics (CLEO), May 2010, Paper CThK7.

[3] Y. Bi, J. Jin, L. G. Kazovsky, "Quasi-Passive Reconfigurable Optical Node: First Experimental Demonstration," in Proc. Conference on Lasers and Electro-Optics (CLEO), May 2012, Paper CTh1H.5.

[4] W. Noell, P. A. Clerc, F. Duport, C. Marxer, N. de Rooij, "Nove process-insensitive latchable $2 \times 2$ optical cross connector for singleand multimode optical MEMS fiber switches," in Proc. 2003 IEEE/LEOS International Conference on Optical MEMS, pp. 49-50, 1821 Aug. 2003

[5] R. Bahuguna, M. Mina, J. Tioh, R. J. Weber, "Magneto-Optic-Based Fiber Switch for Optical Communications," IEEE Transactions on Magnetics, vol. 42, no. 10, pp. 3099-3101, Oct. 2006.

[6] K. Tsunoda, Y. Fukuzumi, J. R. Jameson, Z. Wang, P. B. Griffin, Y. Nishi, "Bipolar resistive switching in polycrystalline $\mathrm{TiO}_{2}$ films", Applied Physics Letters, vol. 90, no. 11, pp. 113501-1-113501-3, Mar 2007

[7] T. Nakagami and K. Nakatsuhara, "Optical routing switches incorporating ferro-electric liquid crystals for use in photonic networks,' in Proc. of the SPIE, vol. 5741, pp. 98-106, 2005.

[8] Y. S. Didosyan, H. Hauser, W. Fiala, J. Nicolics, W. Toriser, "Latching type optical switch," Journal of Applied Physics, vol. 91, no. 10, pp. 7000-7002, May 2002. 